\title{
Wave-particle duality and uncertainty principle: Phenomenographic categories of description of tertiary physics students' depictions
}

\author{
Mengesha Ayene, ${ }^{1, *}$ Jeanne Kriek, ${ }^{2}$ and Baylie Damtie ${ }^{3}$ \\ ${ }^{1}$ Department of Physics, Bahir Dar University, Bahir Dar, Ethiopia \\ ${ }^{2}$ Institute for Science and Technology Education, University of South Africa, Pretoria, South Africa \\ ${ }^{3}$ Department of Physics, Bahir Dar University, Bahir Dar, Ethiopia
}

(Received 23 November 2010; published 3 November 2011)

\begin{abstract}
Quantum mechanics is often thought to be a difficult subject to understand, not only in the complexity of its mathematics but also in its conceptual foundation. In this paper we emphasize students' depictions of the uncertainty principle and wave-particle duality of quantum events, phenomena that could serve as a foundation in building an understanding of quantum mechanics. A phenomenographic study was carried out to categorize a picture of students' descriptions of these key quantum concepts. Data for this study were obtained from a semistructured in-depth interview conducted with undergraduate physics students $(N=25)$ from Bahir Dar, Ethiopia. The phenomenographic data analysis revealed that it is possible to construct three qualitatively different categories to map students' depictions of the concept wave-particle duality, namely, (1) classical description, (2) mixed classical-quantum description, and (3) quasiquantum description. Similarly, it is proposed that students' depictions of the concept uncertainty can be described with four different categories of description, which are (1) uncertainty as an extrinsic property of measurement, (2) uncertainty principle as measurement error or uncertainty, (3) uncertainty as measurement disturbance, and (4) uncertainty as a quantum mechanics uncertainty principle. Overall, we found students are more likely to prefer a classical picture of interpretations of quantum mechanics. However, few students in the quasiquantum category applied typical wave phenomena such as interference and diffraction that cannot be explained within the framework classical physics for depicting the wavelike properties of quantum entities. Despite inhospitable conceptions of the uncertainty principle and waveand particlelike properties of quantum entities in our investigation, the findings presented in this paper are highly consistent with those reported in previous studies. New findings and some implications for instruction and the curricula are discussed.
\end{abstract}

DOI: 10.1103/PhysRevSTPER.7.020113

PACS numbers: 01.40.Fk, 03.65.- w

\section{INTRODUCTION AND BACKGROUND}

Quantum mechanics has become the basic tool of modern physics, and has been successfully applied to a diverse range of fields and applications. Nonetheless, it is widely acknowledged that quantum physics can be intellectually challenging and the courses are academically demanding $[1,2]$. The conceptual changes that the quantum theory has brought about in our understanding of the nature of the subject matter are far reaching and often counterintuitive. The study of quantum mechanics has always presented a huge challenge to the students who study these abstract concepts for the first time. Wave-particle duality causes much confusion, as students' experience has been with things that act like particles, or like waves, but never both. It is usually easier (and more intuitive) to use

\footnotetext{
*Corresponding author. mengesha.ayene@yahoo.com

Published by the American Physical Society under the terms of the Creative Commons Attribution 3.0 License. Further distribution of this work must maintain attribution to the author(s) and the published article's title, journal citation, and DOI.
}

classical physics than the more abstract quantum physics. To assist students to overcome the difficulties of understanding abstract and counterintuitive concepts, it is a common trend to resort to classical images of quantum mechanical effects [3]. The theoretical framework employed by quantum mechanics, however, differs drastically from that of the classical paradigm. Certainly, the transition from a classical to a quantum milieu marks an indisputable revolution in our understanding of the physical world. In view of this disconnect, it is particularly difficult for students to grasp the new concepts [3].

In order to overcome the obstacles in understanding quantum mechanics students have to be made aware of the conceptual difference between classical and quantum physics, as well as the radical conceptual change associated with quantum mechanics. Students therefore have to set aside some preconceived notions that they are brought up with and which stem from their experience of the macroscopic world. However, students who are educated according to the scientific norms found in classical physics and key concepts, such as determinism, causality, etc., can be easily influenced. Having accepted the key concepts of classical physics, they find it difficult to adjust to quantum 
mechanics concepts such as wave-particle duality, uncertainty, probability, etc. [4,5]. Furthermore, in learning classical physics students have already developed visualizable, qualitative mechanical models to understand abstract theoretical concepts used to explain physical phenomena [6]. Such an understanding of classical physics with its use of mechanical models and concepts to explain the physical phenomena contributes to the difficulty of learning quantum mechanics [7] and students' conceptions of quantum entities are essentially simple extensions of classical representations [8]. The reason behind this is that "classical models are persistent and prevalent mental images and very little advancement happens during further teaching" [9].

The presence of several different interpretations of quantum mechanics can make it difficult to categorize students' depictions of wave-particle duality and the uncertainty principle. Especially when discussing students' descriptions of particles, which are delocalized in some interpretations (e.g., the Copenhagen interpretation) but always localized and have a definite trajectory in space in others (e.g., in the Bohmian interpretation). When investigating students' depictions of quantum mechanics topics, it is important to know what interpretations the teachers and students rely on during instruction. Introductory quantum mechanics courses are being instructed in Ethiopian higher institutions (where this study was undertaken) on the foundations of the Copenhagen interpretation. Moreover, instruction at the undergraduate level is highly dependent on textbooks influenced by the Copenhagen interpretation. Thus, this study follows the Copenhagen interpretation of quantum mechanics.

Despite the fact that the learning of quantum mechanics is hindered by students' preconceived classical pictures, an understanding of many classical concepts is a prerequisite to a meaningful understanding of advanced physics such as quantum mechanics and others. When studying quantum mechanics, researchers exemplify two research results to illustrate the impact of student understanding of classical concepts [10]. Typically, physics students first study mechanical waves and then physical optics before moving on to the courses such as modern physics and quantum mechanics. The reasons behind this argument are that the wave properties of matter, wave-particle duality, and atomic spectroscopy make no sense if students do not understand superposition, wave representations, and diffraction [10]. Furthermore, "Quantum physics builds on a classical base, using many classical concepts, variables, and representations. If students are weak on these items, learning of quantum physics may be difficult" [11].

Although this is known, many introductory quantum physics students still face significant challenges when they first learn about the probabilistic features and nonlocal theory of quantum mechanics, which disallows any classical interpretation $[12,13]$. Students' problems in learning quantum mechanics are widespread and may originate from many other sources [2]. To overcome this problem, there is a general agreement among physics education researchers that it is important to gain a better understanding of how students experience, conceptualize and understand key concepts in physics [14]. This was justified as "The key to improving learning is not to be found by searching for 'the best teaching techniques' or 'the vital learning skills'. The truth is much more challenging. The puzzle can only be unlocked by examining what students already know about subject matter and the educational setting in which they learn it" ([15], p. 263). Thus, asking a phenomenographic question, such as, what are the different ways in which students experience, depict, and conceptualize the phenomena under investigation (e.g., the concepts of quantum tunneling), could contribute to an understanding of the challenges. Therefore, in this study we focus on students' depictions of wave-particle duality and the uncertainty principle. Wave-particle duality and the uncertainty principle are features of quantum phenomena that can serve as the foundation in understanding introductory concepts of quantum mechanics. They have been regarded as the most distinguishable features in which quantum mechanics differs from classical theories of the physical world.

\section{STUDENTS' DEPICTIONS OF QUANTUM ENTITIES}

Physics education research suggests that there has been considerable research interest in students' depictions of quantum phenomena over the past 15 years [16,17]. Based on the research reviewed [17], findings in quantum mechanics education research revealed that students are facing difficulties in mastering the subject; for example, "qualitative studies show that many students have considerable problems to depicting a vast array of quantum mechanical topics in an accepted way" ([17], p. 35). Similar findings also reported that students are rather unwilling to learn about quantum mechanical models of the atom. For example, students use a classical atomic model where the fictitious centrifugal forces and electrostatic attractive forces are balanced [18]. According to Mashhadi [18], students' conceptions are mostly in line with the Bohr model of the atom; however, their depictions do not necessarily stem from the Bohr model. In a German pre- and postuniversity study, $63 \%$ of 270 preuniversity students used the "classical orbits depiction" prior to a course dealing with "quantum mechanical orbital theory" and $22 \%$ of the students still used the classical orbits depiction after the pre-quantum-mechanics course [8]. A study in Norway also indicated that students depicted photons as having a dual-nature existence, having both wave and classical particles, whereas electrons were depicted as classical particles only [19]. Unlike the case of the Norwegians, a study in Finland revealed that a classical 
depiction of both electrons and photons was common among a group of physics students [9].

Studies dealing with students' conceptual understandings of topics in quantum mechanics can be found in many developed countries. This is not, however, the case in developing countries. Specific well-documented examples of student difficulties in depicting quantum concepts are often lacking, and the exact nature of the difficulty is often uncertain mainly in a developing country like Ethiopia. Findings from quantum mechanics education literature did not indicate whether students use a consistent depiction of quantum concepts when presented with different situations (e.g., different representations of a single concept) [20]. Therefore, a gap was identified and it was decided to investigate how undergraduate students depict quantum phenomena in Ethiopia. Concepts of quantum mechanics are many and diverse in nature and, consequently, undertaking a comprehensive study of students' depictions in all quantum phenomena was impossible. The research carried out here was aimed at answering the research question: How do undergraduate physics students depict the concepts of wave-particle duality and the uncertainty principle?

The wave-particle duality and Heisenberg's uncertainty principle are phenomena within quantum mechanics that are very interesting to study from a quantum mechanics education researcher's viewpoint. They are topics of quantum mechanics with which students have negligible experience in preparatory school (grades 11 and 12) in the Ethiopian context. Wave-particle duality and the uncertainty principle are features of quantum phenomena that can serve as a foundation in understanding quantum mechanics $[9,21]$. Furthermore, these concepts are assumed by many to be some of the difficult and counterintuitive topics of quantum mechanics that have recently started to attract the interest of physics education researchers [2].

\section{PURPOSE OF THIS STUDY}

The goal of the study was to explore the ways in which undergraduate physics students depict wave-particle duality and the uncertainty principle in qualitatively different categories of description. In this paper, we are interested in exploring the variation in students' depictions of waveparticle duality and the uncertainty principle. The purpose of the study was not to describe the individual students' responses. Individuals are seen only as contributing fragments of data to a given category of description. In other words, the categories of description represent the variation in ways of depicting the phenomenon under investigation at the collective level. This focus on the collective variation made it suitable to choose phenomenography, a qualitative research methodology, as our analytical approach. Phenomenography is based on the essential assumption that it is possible to describe the ways in which a given set of students experience, conceptualize, and understand a particular phenomenon in a limited number of qualitatively different categories of description [22]. In phenomenography, the categories of description are distinguished from one another in terms of the presence or absence of specific essential attributes of the phenomenon under investigation, and it is in this sense that the categories of description are said to be qualitatively different. Thus, analysis of data is based on the phenomenographic approach. The categories of description (outcome spaces) are analytically drawn from all the collected data, the students' in-depth interviews [22,23], and from the students' written comments to the interview questions [24]. A phenomenographic data analysis sorts these perceptions, which emerge from the data collected, into specific categories of description that are structurally linked to one another. The categories are not determined in advance and the researcher must strive to describe the experience of the participants. Particular to this study, we report the qualitatively different ways of depictions that emerged from the responses of undergraduate physics students when asked to reveal their depictions of the wave-particle duality and the uncertainty principle.

\section{RESEARCH SUBJECTS AND CONTEXT OF THE STUDY}

Currently university programs naturally reflect the importance of quantum mechanics in modern physics courses. In Ethiopian universities, for example, an essential qualitative introduction with some quantitative formalism to fundamental quantum mechanical ideas is given starting from the first year in the Bachelor of Science physics program. In the second- and third-year physics program, basic quantum mechanics and its most important applications are studied in detail. For the purpose of this study, 25 second-year students (21 male and 4 female) were selected from students who are following a three-year degree physics course presented in a predominantly traditional manner at Wollo University, one of the higher education institutions in Ethiopia. The participants in the study had completed a Modern Physics course which comprises 45 lecture hours in their first year and Quantum Mechanics I, which comprises 45 lecture hours in their second year before they participated in this interview session (see Table I). Phenomenographic investigation inclines to use what Patton termed as "maximum variation" sampling strategy [25]. It is a strategy for purposeful sampling as the researcher intentionally selects research participants whose insights are related to the central question but who also provide a broad range of views (maximum variation).

The participants for the interviews were selected based on the results of their first-year final grades of the Modern Physics course in order to obtain a cohort with a cross section of depictions or variations of descriptions of the subject under study. For this selection process the students' final grades were grouped in three levels, as C (low), $\mathrm{B}$ (medium), and A (high). Out of the total population of 38 students, 27 students scored $\mathrm{C}, 7$ students scored $\mathrm{B}$, and 
TABLE I. Summary of the two Quantum Physics courses completed by students who were recruited for interviews, including the descriptions of the progression topics, total number of lecture hours devoted, and reference books used for each Quantum Physics course.

\begin{tabular}{|c|c|c|c|}
\hline Quantum Physics course & Description of topics & $\begin{array}{l}\text { Number of lecture } \\
\text { hours devoted }\end{array}$ & $\begin{array}{l}\text { Recommended } \\
\text { reading }\end{array}$ \\
\hline Modern Physics & $\begin{array}{l}\text { Principle of Special Theory of Relativity; Michelson- } \\
\text { Morley experiment; Galilean transformation; Lorentz } \\
\text { transformation; length contraction; time dilation; relativistic } \\
\text { momentum and energy; blackbody radiation; photoelectric } \\
\text { effect and Compton effect; matter waves; uncertainty } \\
\text { principle; and atomic structure. }\end{array}$ & 45 & {$[36,37]$} \\
\hline Quantum Mechanics I & $\begin{array}{l}\text { Limitations of classical mechanics; origin and development } \\
\text { of quantum mechanics; review of modern physics (particle } \\
\text { aspect of radiation, wave aspect of particles, particles versus } \\
\text { waves, Heisenberg's uncertainty principle, probabilistic in- } \\
\text { terpretation, wave packets); mathematical foundation of } \\
\text { quantum mechanics; operator algebra; Schrödinger and } \\
\text { Heisenberg equations; and the harmonic oscillator. }\end{array}$ & 45 & {$[38,39]$} \\
\hline
\end{tabular}

only 4 students scored A. Only 14 students were randomly chosen from the C-scored (low) group, and all the other students who scored B and A were purposefully included in the sample. The selected students were approached and invited to volunteer for the interviews. All the students agreed to participate and were assured that they were free to withdraw at any time and that their participation or nonparticipation would have no effect on their grades. The interviews were carried out over a 2-week period, following the 45-hour lecture course of formal instruction in Quantum Mechanics I in their second year.

\section{DESIGN AND PROCEDURE OF THE STUDY}

A research design provides the framework that identifies the type of information to be collected, its sources, and the collection procedure. The method selected supports the qualitative research approach of phenomenography. In phenomenography, the semistructured in-depth interview $[22,23]$ is regarded as the preferred data-collection method, with an emphasis on providing open-ended questions that encourage the participants to express their own perspectives on both the wave-particle duality and the uncertainty principle.

In this study semistructured interviews were used, in which specific questions were prepared, but any unexpected lines of reasoning were also followed. A number of conceptual surveys covering various quantum mechanics domains have been developed in recent years [21,26-30]. The specific interview questions were constructed based on the review of previous conceptual survey instruments [21,26-30] and by analyzing the syllabi of the two quantum mechanics course structures (Modern Physics and Quantum Mechanics I) (see Appendix). The analysis was conducted as follows: the topics taught in the two undergraduate quantum mechanics courses were identified and the frequencies with which these topics appear in the two courses were counted. Finally, the predetermined interview questions were built around this information, as it was hoped that both the review and analysis of the syllabi would provide valuable information concerning the content and form of interview questions which could be used in the study. In the in-depth interviews, each interview session concentrated on the main features of the wave-particle duality and the uncertainty principle (e.g., the properties of particles and waves within the contexts of classical and quantum physics). Moreover, a series of simulated figures presenting the two concepts (e.g., the double-slit experiment) were shown and students were asked to give explanations and written answers based on their experiences and understandings. They were encouraged to "think aloud" whenever they were to respond to pictures and diagrams.

The phenomenographic interviews were designed to obtain a qualitative description of the conceptual understanding of the interviewee. The research interviews were conducted individually with the interviewee's consent and were digitally audio recorded. The interviews were conducted in English and took between 35 and 65 min. It was important to spend time in conversation with the interviewees prior to conducting the formal interview and the audio recording thereof to put the students at ease and to offer them a safe and comfortable atmosphere in which to speak. The interviews were held in an office or a classroom with closed doors to ensure privacy. All the individual depictions of the two concepts (wave-particle duality and the uncertainty principle) under investigation were collected into a common "pool of experiences" from which "categories of description" were constructed that correspond to the possible qualitatively different ways of depicting the concepts under investigation. 


\section{ANALYSIS AND CATEGORIZATIONS OF RESPONSES}

In phenomenographic analysis, the process is iterative and comparative and involves the continual sorting and resorting of data and ongoing comparisons between data and the developing categories of description [22]. The interviews were transcribed verbatim from the audio record and used in conjunction with the students' written comments in the form of graphs, diagrams, and pictures (e.g., students' response to the double-slit experiment). Video recordings were not done, but gesture information was included in the verbatim notes. Despite its vital role in discerning students' understanding of physics concepts, video-recorded gesture information data were not included in analyzing students' depictions in the study. However, it is unlikely that the overall results or descriptions of students' depictions have been affected by the exclusion of gesture information. During the analysis the information gleaned from the transcripts of interviews was sorted and grouped together into the two concepts (concept 1 is waveparticle duality and concept 2 is uncertainty principle). Further analysis followed in each concept category by selecting segments of text which were regarded as relevant to the basic concerns of the study. This selection process consisted of recording key words, phrases, and passages, which the participants themselves had repeated or had indicated as being important.

A set of text-based categories was developed which paraphrased or generalized the text itself and contained the key characteristics of each category. For example, many of the participants depicted the interaction of light with the screen surface on the photographic plate to be both wave- and particlelike nature of light. This is illustrated by the following quote: it (light) is behaving like a wave and a particle. Again, we organized the text-based categories into the final principal categories. In each text-based category a variation existed in the way it is described, and thus, by identifying the variations it assisted in identifying the categories of description. After the areas of variation were found, the categories of description could be determined. Consequently, all the data were divided into the specific category of description and three categories of description for concept 1 and four categories description for concept 2 (see Fig. 1) were created. The three principal categories of description of concept 1 (wave-particle duality) are classical description, mixed classical-quantum description, and quasiquantum description. The four principal categories of description of concept 2 (uncertainty principle) are uncertainty principle as an extrinsic property of measurement, uncertainty principle as measurement error or uncertainty, uncertainty principle as measurement disturbance, and uncertainty principle as a quantum mechanical uncertainty principle. Figure 1 illustrates the graphical layout of the two concepts and the categories of description of students' depiction of the concepts under investigation.

Taking these categories into consideration all the interview transcripts were reexamined to determine whether these categories were sufficiently descriptive and indicative of the data. Table II outlines the students' depiction of the three principal categories of description for waveparticle duality and the text-based categories (key characteristics of each category).

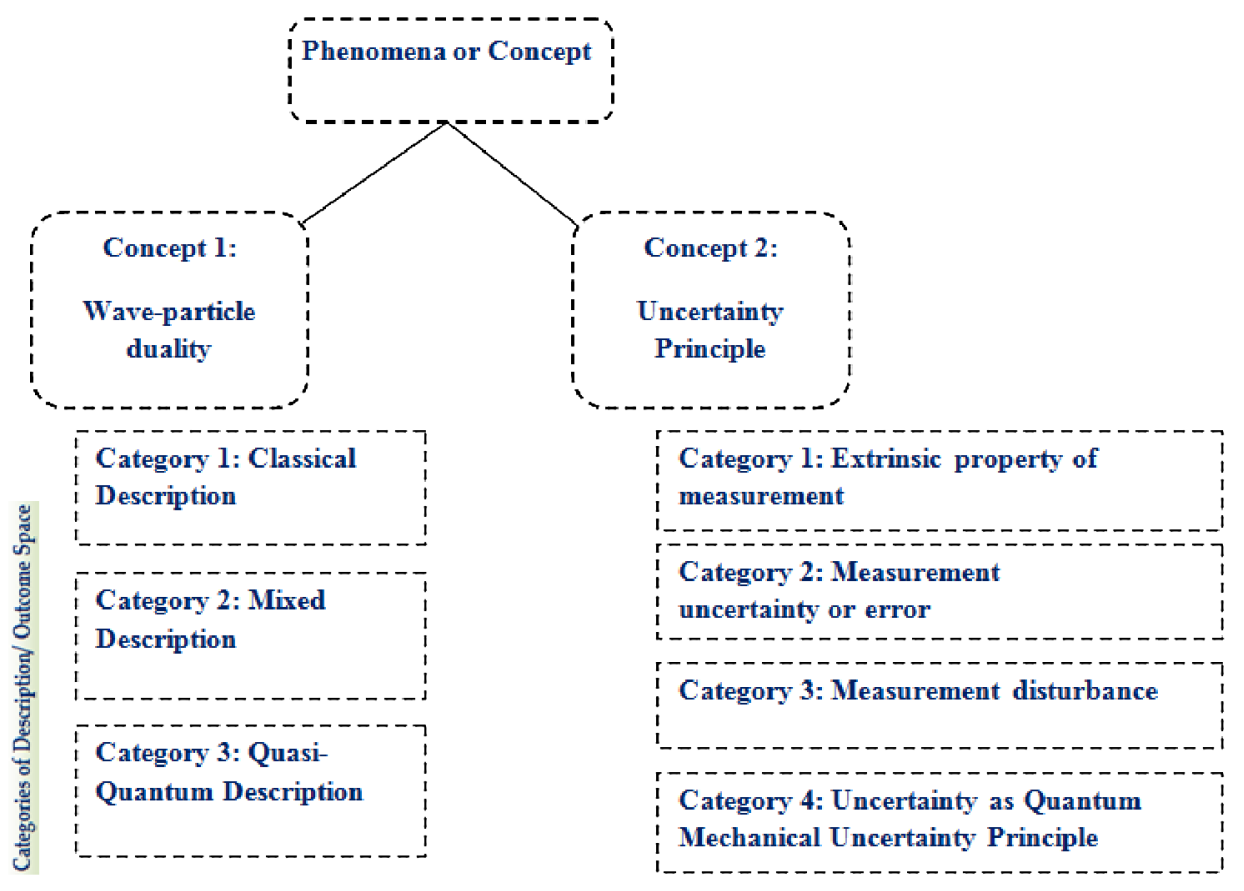

FIG. 1 (color online). The graphical layout of the two concepts and categories of description. 
TABLE II. Students' depiction of concept 1: wave-particle duality.

\begin{tabular}{ll}
\hline \hline Category & Text-based category (examples of key characteristics from students' responses) \\
\hline Classical description & Describing all objects either as a particle or as a wave as if they are mutually exclusive. \\
& Particles are described as localized, compact, hard, and massive objects. \\
& Visualizing particle as a billiard ball which carries energy and momentum. \\
& Wave characteristics are described as a simple disturbance. \\
& Understanding that the photoelectric emission is due to continuous absorption of photons; intensity of \\
& a light source is considered as the determining factor for the emission of an electron during the \\
& photoelectric effect. \\
& Interpreting the interaction phenomena of electrons with the screen (in the double-slit experiment) as \\
& the wave nature and particle nature of an electron; describing the interaction phenomena of the \\
photon with the screen as the wave nature and particle aspect of light; describing light as a wave. \\
Describing the formations of interference fringes on the screen as a function of the intensity of the \\
source (number of photons or electrons).
\end{tabular}

Mixed description

Macroscopic objects are seen as objects having properties of classical waves.

Photons are seen as objects having properties of classical particles and waves.

Electrons are described as objects that behave like localized objects but do not possess the property of the position.

A beam of photons is conceived as objects possessing an energy $h \nu$ and momentum $m v$ (mass $\times$ velocity).

Considering photons as classical particles. That is perfectly localized point objects containing the $x$ and $y$ coordinates.

Referring to textbook notes of experimental explanation to describe particles such as electrons as having wave property.

Theoretically both macroscopic and microscopic objects are depicted as objects having wave properties.

Formation of interference fringes are correctly described as a wave property of objects but as a function of intensity (e.g., if intensity decreases the interference fringe on the screen also decreases). Every photon and electron passing through the slit is expected to form an interference fringe on the screen.

Quasiquantum description In the double-slit experiment electrons are expected to form an interference fringe and increasing the speed of elections will be expected to narrow interference maxima and minima.

The de Broglie wavelength is considered as key idea to describe the wavelike property of matter. Interference and diffraction are used to characterize the wavelike property of electrons and photons. The interactions of photons with the screen are described as a particle property; the wavelike nature of light is demonstrated by interference and diffraction properties.

Emission of electrons in the photoelectric experiment is interpreted as a function of frequency rather than intensity.

Electrons are described as quantum entities having typical wave phenomena such as superposition and interference but also trajectories to locate their path; the particle nature of the photons is seen as quantum properties having different properties than classical particle properties.

Individual photons are detected as localized particles;

Photon momentum is described in terms of wavelength and Planck's constant.

Table III outlines the four principal categories of description identified and the text-based categories (key characteristics of each category) for students' depictions of the uncertainty principle.

By using the text-based categories depicted in Tables II and III, the principal categories were further constructed by identifying illustrative quotes from the responses received during the in-depth interviews. In the following section, excerpts of answers to interview questions are presented as illustration of the construction of the different categories. Nevertheless, it has to be understood that the categories are defined in terms of the students' responses to all the interview questions, not just the one example given here; this example is given for just illustrative purpose.

\section{A. Students' depictions of wave-particle duality}

In the analysis of the transcripts three qualitatively different ways were identified in which students depict and experience the concept of wave-particle duality. These various ways of depicting the concept of waveparticle duality ranged from the classical deterministic picture of quantum entities to quasiquantum views. 
TABLE III. Students' depiction of concept 2: uncertainty principle.

Principal category Text-based category (examples of key characteristics from students' responses )

Extrinsic property of measurement

Uncertainty is described as a measurement error due to external effects from outside such as thermal agitation, noise, vibration, the surrounding contacts, etc.

Uncertainty is understood as a failure of the experiment due to lack of resolution, reliable technology, or equipment.

Modern scientific devices are thought to measure repeated errorless measurements of the variable which can always give precisely the same value.

Measurement error or uncertainty

Describing $\Delta x$ as an uncertainty or error varies from the exact value; if the minimum error is known in position measurement the minimum error in the momentum measurement is also known.

Uncertainties in the measurement of observables are subject to only technical or random errors. Uncertainty is described as an error that occurred during measurement.

Uncertainty is seen as some limitation or error of the instrument.

No measurement is found without at least minimum error.

Uncertainty is depicted as measurement errors due to the lack of skilled technicians who measure without a small error.

Uncertainty is understood as measurement errors related to mistakes or wrong measurements.

Measurement disturbance

Measurement of a particle's position greatly disturbs its momentum.

Regarding the uncertainty relation as a consequence of the disturbance of the measurement process.

Describing the uncertainty principle as a consequence of unavoidable disturbance to the system resulting from the act of measurement.

The position of a particle is well defined when one specifies a suitable experiment that allows the position of a particle to be measured.

The momentum of a particle changes, due to a position measurement.

Uncertainty as a quantum mechanical uncertainty principle

The position and momentum cannot be simultaneously and accurately measured.

Uncertainty does not work practically since $\Delta x \Delta p \geq \hbar / 2$ and $\hbar$ is very small.

$\Delta x$ is the variation during measurement.

Uncertainty is described as an intrinsic property of measurement.

$\Delta x \Delta p$ is described as an uncertainty relation which illustrates more accurately the measurement of the position (minimum $\Delta x$ ) the greater the uncertainty of momentum (maximum $\Delta p$ ). Uncertainty relation is described in terms of Planck's constant. That is, the smaller $\Delta x$, the larger $\Delta p$ has to be, and the relation always holds true.

\section{Category 1: Classical description}

The term "classical description" is used to indicate that students' described quantum entities in terms of the classical picture of particles and waves. In this group students depict microscopic particles such as electrons as localized, compact, hard, and massive objects that carry energy and momentum. The wave nature of photons is also characterized by classical wave properties as a simple disturbance, like water wave moving in an extended medium and as a diffusing of object in space and time which has wavelengths and frequencies. These are examples of student responses to the following interview question.

In Modern Physics and Quantum Mechanics courses we say electrons, protons, and photons behave like waves, as well as like particles. What would you say are the simplest "particlelike" and "wavelike" properties that one of these things (electrons, protons and photons) could show?
Yes um ... um ... to me particles are small material objects even all objects and may be a ball an electron etc. that are characterized by their one position and second motion or momentum (momentum is mass times velocity). They have also energy. And these things could show to say particle. In wavelike ... wave is a disturbance that propagate or diffuse from place to place. It has frequencies and wavelength.

Moreover, students in this group also preferred the classical description of particles when they were asked to explain the photoelectric effect and the path of the electron in the double-slit experiment.

To illustrate their reasoning they would indicate that an electron would keep on absorbing energy at a continuous rate until it gained a sufficient amount, then it would leave the metal surface. Furthermore, they relate the emission of electrons to the intensity of light (the number of photons). 
When students holding this classical description view were asked the question "what will happen to the path of the electron if the slit width increased?" they applied a line to map the path of electrons from the source to the screen. When students were shown a simulated figure of a doubleslit interference and diffraction pattern with different number of photons from the source and were asked to predict what would happen on the screen, they depicted that the interference fringes in the screen would disappear if the intensity of photons (the number of photons) reaching the screen diminished. In addition, these students described the patterns on the screen quite differently for electrons and photons when the source in the double-slit experiment was changed to electrons.

Generally, students in this group grouped quantum entities as a particle or a wave as if they were mutually exclusive. These classical descriptions were also found in the other categories of description. However, the extent to which it was used decreased going down the hierarchical structure of categories from classical to quasiquantum categories of description.

\section{Category 2: Mixed description}

The key characteristics or text-based categories are presented in Table II. When transcribing interviews and analyzing questionnaires, it was found that students in this group incorporated the classical picture of reasoning into the quantum view. Students' depictions indicated that they frequently mixed up the quantum description with the dominant classical pictures of description, resulting in confused mental images. For example, students depicted that a beam of photons possesses an energy $h \nu$ and momentum $m v$ (mass $\times$ velocity). However, its particlelike characteristics were discerned by these students as localized point objects containing the $x$ and $y$ coordinates relative to the source. The following statements are examples of this category.

In quantum mechanics we say that light behaves like a particle, as well as like a wave. What is meant by that statement?

It is true. That means light photons show interference and diffraction in experiment ... this is like wave. Again it acts like particles.

What is meant by "it acts like particles"?

Umm ... you know particles are objects It is possible to indicate polar coordinates or others position vector. It has mass velocity, position so possible to calculate its momentum by calculating $M \times V$. I think so.

This comment illustrated that this student experienced the particle nature of photons as being localized in coordinates and having mass. Moreover, students in this category also depicted photons as a wave and a particle while the electrons and protons were perceived as particles only. Students' depictions of the wave nature of electrons, as well as the interference fringe pattern in the double-slit experiment, appeared to revert to a classical picture when the nature of the experiment was changed (e.g., the electrons were sent at one time; i.e., each electron was sent only after the previous electron had reached the screen). In general, according to these students' descriptions, the interference pattern they observed in the simulated figure disappears if the experiment is performed in such a way that on average only one electron at a given time arrives at the screen.

\section{Category 3: Quasiquantum description}

In this category student depictions of the wave-particle duality were predominantly following the standard quantum views of reasoning. Nevertheless, students sometimes considered quantum entities as classical waves and particles. They have understood that microscopic particles, such as electrons and protons, display wave behavior. Students in this category took things even further by suggesting that this wave-particle duality is not restricted to microscopic particles, but must be universal: all material particles should also display dual wave-particle behavior. That is, students understood that the wave-particle duality present in microscopic objects must also occur in all matter. The arguments forwarded for their description were supported with mathematical explanations of the de Broglie wavelength with Planck's constant $h$. In particular, students used the de Broglie wavelength description to explain why the wave associated with material particles was not discernible, even though macroscopic material particles display wave properties. The following question and student's answer illustrate this perception.

You know about water waves, surface waves, micro waves, sound waves and light waves in your Wave and Optics course. In 1924 Louis de Broglie proposed that electrons and other macroscopic entities are also waves. What do you mean when you say "something is a wave in quantum physics"?

Yes of course in this course (i.e., quantum mechanics I) and in modern [...] we learned wave-particle. Light is a wave and a particle. The de Broglie wavelength $=h / p$ umm [...] this means all matter have wavelength but small. So indicating matter discern wavelike nature in the same way microscopic objects do.

Students depicted that the interference pattern obtained from the double-slit experiment in the simulated figure indicated that electrons display both particle and wave properties. The problem students faced with this experiment is that like in classical physics, they saw particles following trajectories as if they were conventional objects, their trajectories accurately determining their path. In general, these students based their explanations correctly on the quantum view of particles and waves, but sometimes they incorporated the classical view of particles and waves. 


\section{B. Students' depictions of the Heisenberg uncertainty principle}

The following four categories of descriptions were used to describe students' depictions of the concept uncertainty principle as derived from the phenomenographic data analysis, namely, extrinsic property of measurement, measurement error or uncertainty, measurement disturbance, and uncertainty principle.

\section{Category 1: Extrinsic property of measurement}

The key characteristics of this category indicated that students depicted the concept of uncertainty principle as being related to their daily measurement issues (Table III). Particular to this category, students described uncertainty as a measurement error due to an external effect such as thermal agitation, noise, vibration, the surrounding contacts, etc. Furthermore, they described uncertainty as a lack of resolution, and technology or equipment not being good enough. In order to further investigate students' depiction of the concept of uncertainty, one interview question from previous research was adopted here [26]. "Consider a "measurement" involving catching your university bus in a quantum mechanical world. Your timetable says that the bus will arrive at 2:30 am. Heisenberg would say that the time of arrival must have an associated uncertainty. What does he mean by uncertainty?" The following student quotes are illustrative of how a student perceives and understands uncertainty.

Yes I think the problem is the car may be don't reach at time. Many problems are, for example, the bus may have accident, umm if the road is not safe I mean closed due to construction, etc. for example, the driver may ill. In our university this common so the bus may late.

\section{Category 2: Measurement error or uncertainty.}

The analysis indicated that students in this class expressed the concept of the uncertainty principle as an uncertainty in classical physics. This category resembles that of the previous category, in that there is a focus on the measurement error due to the limitation of the instrument. For example, students described the meaning of $\Delta x$ as an error or uncertainty that departs from the exact value because of mistakes, wrong measurements, or instrument limitations. The same question, "catching your university bus in a quantum mechanical world," was put to another student. An excerpt follows that gives evidence of the student's viewpoint.

The uncertainty in this case umm .... For me there are many problems. My clock reading is different from the driver. For example the driver read his watch wrongly. For example the drive should start from "Dessie" at 1:35 to reach here "Kombolcha" at 2:30. But they (drivers) carless and read the 1:25 as 1:35 at "Dessie". This is error. So not reach at time. I think this is
It can be seen from these statements that this student understands the uncertainty principle as measurement error or uncertainty, as illustrated in the following excerpt:

Can you describe mathematically the Heisenberg uncertainty relations?

Yea $\Delta x \Delta p \geq \hbar / 2$.

What do we mean by $\Delta x$ ? What is $\Delta x$ ? What is the meaning of $\Delta x$ ?

Uncertainty

What is uncertainty?

It is an error. I mean you have [...] yes real and measured value I think is the difference of the two. By the way no measurement is found without minimum error. Efficiency never $100 \%$ no technician measure without a small error. It is that

This student mathematically expressed the uncertainty principle as is proven by the answer in the above excerpt, but he perceived that quantum uncertainty implies measurement error.

\section{Category 3: Measurement disturbance}

As with the previous categories, this category contains expressions of uncertainty where it is compared or contrasted with measurement uncertainty. In this category, students link the idea of uncertainty principle to the term measurement disturbance. These ideas point to the contrasting meaning of the term. This is conveyed in the following statement by a student.

For me according to uncertainty principle ... uncertainty is I mean nothing measured correctly. Because, for example, by uncertainty principle if measure the position of the ball, the momentum will be disturbed. Impossible. If there is no disturbance I do not know it may be possible.

\section{Category 4: Uncertainty principle as a quantum mechanical relation.}

In this category, most of the features of the uncertainty principle are discerned with a correct line of reasoning. Students understood that in quantum mechanics the idea of uncertainty was proposed in response to the wave-particle duality of microscopic objects. Despite their responses being direct memorization of textbook definitions or definitions stated by teachers, students discerned the uncertainty principle in the context of quantum mechanics in this category. The following student quotes are illustrative of why a student's description is grouped in this category.

In quantum physics, how does one know whether it is possible to have complete simultaneous knowledge of two specific properties of a system, say " $x$ " and/or " $p$ "?

According to the definition of Heisenberg uncertainty it is impossible. By the way I read a book it says the position and momentum cannot be defined cannot be measured accurately at the same instant of time.

The way in which students had presented their depictions of the uncertainty principle appeared so vague that it 


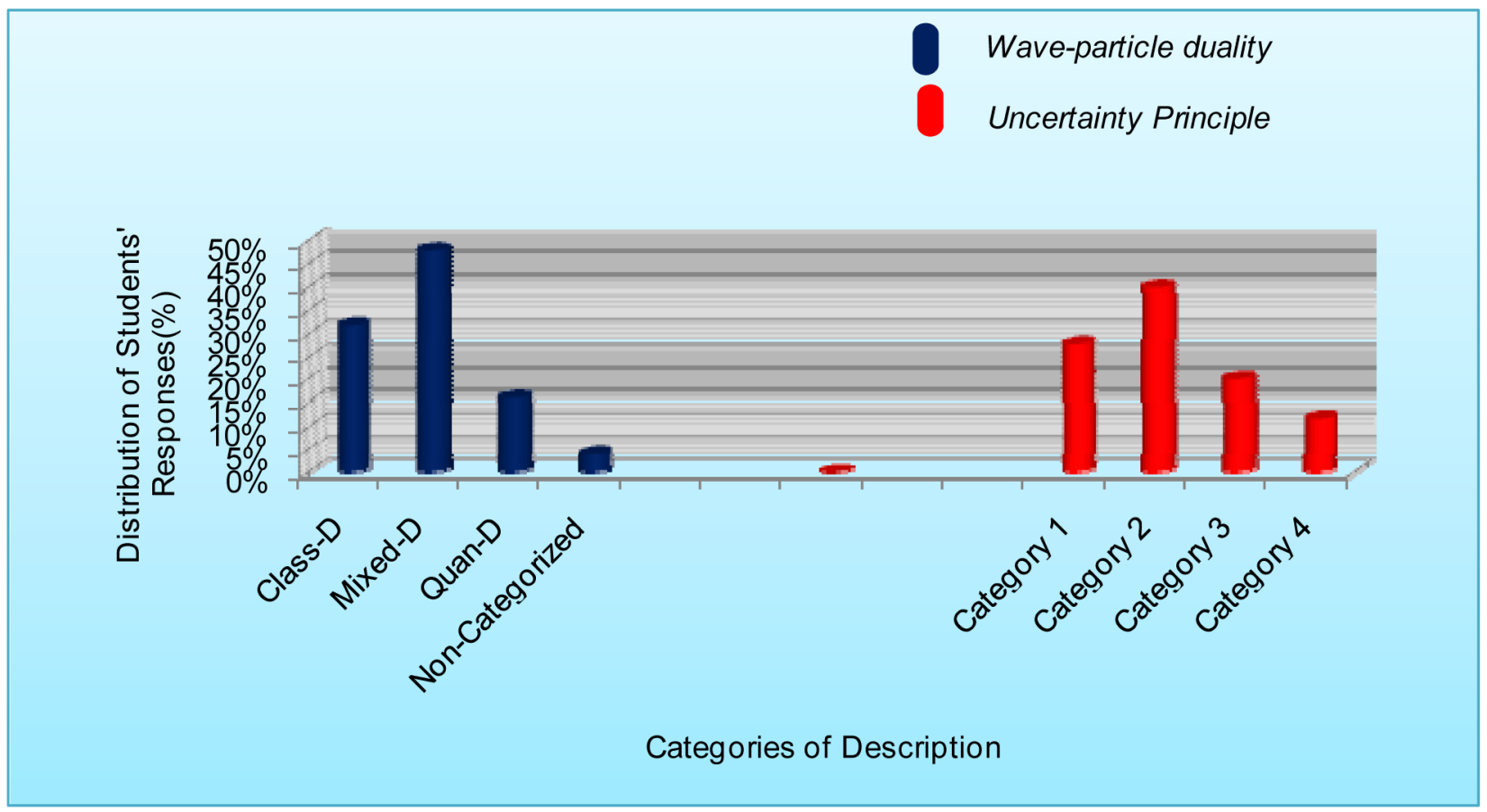

FIG. 2 (color online). Distribution of students' responses in each category for the concepts wave-particle duality and the uncertainty principle. Codes: classical description (Class-D), mixed description (Mixed-D), and quasiquantum description (Quan-D).

was not clear whether these had stemmed from a system's wave function or from any quantum mechanics postulate.

In an effort to clearly understand the meanings in his expression, this student was asked the following questions.

Cannot be measured what does it mean? Do you mean failure of experiment, lack of scientific equipment or what?

No [...] long pause ... no I remember the thing is not related with measuring equipment. We learned that it is natural.

In the analyzed interview, the students demonstrated an understanding of the uncertainty principle in the context of quantum mechanics by indicating that it would not be possible to know the values of both position and momentum with arbitrary accuracy. Students' responses asserted that a quantum system simply does not possess a definite value for its position and momentum at the same time. As shown in the responses in various categories given in Table III, students' depictions were that according to quantum mechanics, the more precisely the position (momentum) of a particle is given, the less precisely can one say what its momentum (position) is; yet the fact remains that the students' depiction is a simplistic and preliminary formulation of the quantum mechanical uncertainty principle for momentum and position. The interview responses confirmed that students are recognizing uncertainty as a new concept which is different from everyday language; however, in conclusion based on students' depictions of the uncertainty principle, it seems that students simply memorize the textbook definition or teachers' lecture notes and apply this knowledge to the world of quantum mechanics.

\section{Distribution of students' answers in the categories}

Figure 2 shows the percentage distribution of the students' responses in each category for the concepts waveparticle duality and the uncertainty principle.

In the presentation of wave-particle duality as shown in Fig. 2, 80\% (20) of the students' depictions fall into the categories mixed description and classical description. Students' depictions in the category quasiquantum description comprise $16 \%$ (4). Only $4 \%$ (only one) of all participating students' excerpts could not be categorized in terms of the three categories discerned. Regarding the concept of uncertainty principle, students' distribution across the first three categories (category 1, extrinsic property of measurement; category 2, measurement error or uncertainty; and category 3, measurement disturbance) accounted for $88 \%$ (22) of the sample students' interview excerpts. Students' depiction of category 4, namely, the uncertainty principle as a quantum mechanics uncertain principle, only accounted for $12 \%$ (3) of students.

\section{DISCUSSIONS}

Our aim in this paper was to explore students' depictions of the concepts of wave-particle duality and the uncertainty principle. The study was aimed at establishing the variation in the ways students depict the phenomena under investigation. Therefore, we analyzed the interview data using the qualitative phenomenographic approach [31]. Since our main goal was to sort the depictions, which emerged from the data collected, into specific categories 
of description, students' depictions of these two concepts were discerned and the structure of their descriptions was constructed. The group of participant students interviewed had varying depictions of the concepts of wave-particle dualism and uncertainty principle, and we have been able to categorize $96 \%$ of their responses into a small number of qualitatively different ways of depictions. These categories with their key characteristics are summarized in Tables II and III. A paradigmatic description of the categories with excerpts from both the interviews and the written comments is presented to illustrate the findings.

As illustrated in Fig. 2, the classical picture of description and the mixed classical-quantum description which accounts for $80 \%$ of the students' responses are found to be inadequate to describe quantum mechanics and are influenced by the classical deterministic picture of descriptions. For example, students in both categories are aware of the fact that the position of a quantum particle is not described by a probability distribution but by a single sharp value. The third category quasiquantum description for the concept of wave-particle duality is relatively quantumlike. For example, students in this category used typical wave phenomena such as interference and diffraction that cannot be explained in a classical particle picture for depicting the wavelike properties of electrons. However, in some cases students in this category still thought in terms of the classical and mixed picture of reasoning.

In describing the uncertainty principle most of the participating students fall into the three categories (extrinsic property of measurement, measurement error or uncertainty, and measurement disturbance) that accounts for $88 \%$ of the sample. This is highly influenced by the classical ontology of measurement error. Only $12 \%$ of the participants' expression described the uncertainty principle based on the standard interpretation in quantum mechanics. Although these students experienced the uncertainty principle based on the standard interpretation, they described it by memorizing textbook definitions and examples. Unlike previous studies, we found that no participant students failed to express the mathematical formalism of the uncertainty principle. This could be due to the fact that the mathematical uncertainty principle appears only in quantum mechanics courses and it has no analogue in classical physics.

\section{WHAT DO THE RESULTS TELL US?}

This investigation has identified the wide variation in the ways students describe and depict their conceptual understanding of the quantum entities wave-particle duality and uncertainty principle. The phenomenographic data analysis revealed that students often depend on the mechanical and deterministic paradigm of classical physics for the depiction of the quantum physical system. A good illustration of this observation can be found in the students' depiction of the particle aspect of radiation, the wavelike aspect of particle, and students' explanation of the uncertainty principle. In conclusion, it is noticeable that after quantum mechanics instruction students still seem to hold on to a deterministic world view. Many students who participated in the study were unable to relinquish their initial knowledge stated based on classical physics instruction and adopt the quantum mechanical framework in describing atomic and subatomic phenomena. For instance, these students make use of the words "position," "momentum," "particle," and "wave" in quantum mechanics as if it were classical physics. After formal quantum mechanics instruction, students still apply mixed classical and quantum descriptions when dealing with a quantum mechanical framework. In view of these findings, we conclude that quantum mechanics traditional teaching methods favor inconsistent learning and thus fail to provide proper understanding.

Despite the inhospitable results in our investigation, the findings presented in this paper are also consistent with those of previous studies in different contexts and countries [9,32]. For instance, Mannila et al. [9] addressed students' conceptions on quantum concepts such as wave- and particlelike properties of quantum entities using an approach based on qualitative problem solving. In their study, students, who were chosen from two intermediate-level courses on quantum mechanics were shown a series of figures presenting the gradual formation of an interference pattern. They were then asked to give written answers to eight open-ended questions with regard to the observed phenomena in the double-slit experiment. From the responses of the students' written answers, Mannila et al. [9] were able to identify various models and categories of description, namely, particle ontology based, trajectory based, statistical, and quasiquantum models. The researchers found that the responses were dominated by classical pictures and trajectory based reasoning, as described by categories which are quite similar to the categories of descriptions depicted by the students participating in this study.

Our results are also similar to those obtained at the University of Sydney in Australia. A research study in Australia by Johnston et al. [32] reported that the university students who participated in a study had difficulty describing what characterizes a particle or a wave. In the study, these students also found it difficult to distinguish between quantum indeterminacies and measurement uncertainties. In another study in Australia, Fletcher and Johnston [16] explored the conceptions that students hold after completing their first year of tertiary studies in quantum mechanics. In order to analyze and interpret the data, the researchers adopted a phenomenographic analysis supported by content, context and correctness analyses. Their study found clear evidence that the majority of students did not understand the key concepts quantum mechanics covered in their tertiary physics course which corroborates our 
findings in this study. In England, Ireson [33] reported on the concepts that undergraduate physics students hold regarding quantum phenomena using a questionnaire for data collection and two multivariate techniques for analysis. A cluster analysis of participating students' conceptions in that study generated three distinct clusters, which were named quantum thinking, intermediate thinking, and mechanistic thinking. This is in agreement with our findings reported in this paper. In the Norwegian study, the findings were quite similar to those of our investigation, as many students also depicted photons as having both wave and particle properties, the double-nature phenomenon, while electrons were depicted as classical atomic particles only [19]. There is also a large body of physics education research in quantum mechanics that shows students have many alternative conceptions about quantum physics in the level of modern physics and quantum mechanics [34,35]. These alternative conceptions are often about the quantum mechanical conception itself and about utilizing this conception to infer what should happen in a given situation. Students in these studies often overgeneralize their intuitive notions from the classical physics to the quantum physics, which can lead to erroneous inferences. This was also found in our study; therefore, with regard to quantum mechanics instruction at the undergraduate level, the present study strengthened the findings reported in the previous studies in that the highly formalized traditional teaching methods lead to a rather fragmentary "shallow learning." In light of the conceptual difficulties identified in this study and in previous studies, it is deemed important to go beyond traditional instruction to allow students to develop a deeper conceptual understanding of quantum mechanics.

\section{IMPLICATION FOR TEACHING AND LEARNING}

The categories of description now provide a platform for application to teaching practice. Because of the hierarchical nature of the categories, we would obviously select the majority of our students to hold at least a quasiquantum depiction of the concepts under investigation by the time they finish an undergraduate introductory quantum mechanics course. This may be achieved by designing curricula and pedagogical approaches that will allow students to develop those understandings. The most important step is to recognize that our students in an undergraduate introductory quantum mechanics course hold different views on the nature of wave-particle dualism and uncertainty principle. By the time physics students enter a quantum mechanics course, they have already accepted the concepts of classical physics, that is, the fundamental concepts of classical particle, waves, and uncertainties that are usually easier to use and to understand and are very convincing. Having accepted the fundamental concepts of classical physics, they find it difficult to adjust to those of quantum mechanics such as wave-particle duality, uncertainty, probability, etc. [5]. This knowledge gap needs to be addressed in our teaching, and an important first step is to take opportunities to introduce students to the full range of differences for studying the properties of particles and waves within the contexts of classical and quantum physics. The student can at this stage be introduced to various ideas, terms, and words such as particles, waves, and uncertainties (which are mutually exclusive in the classical context) when used in quantum mechanics. In particular, the uncertainty principle and wave-particle duality should be presented not just as theoretical ideas but in light of experiments and/or computer simulations for illustrations. These experiments and/or computer simulations could be demonstrated during a lecture with students partaking in a discussion, or they could be executed as a tutorial with conceptual questions relating to wave-particle duality and the uncertainty principle.

\section{APPENDIX: INTERVIEW QUESTIONS}

See separate auxiliary material for interview questions based on the conceptual survey and quantum mechanics courses.
[1] T. Kallio-Tamminen, Quantum metaphysics. The role of human beings within the paradigms of classical and quantum physics, Ph.D. thesis, University of Helsinki, 2004.

[2] D. A. Muller, Designing effective multimedia for physics education, Ph.D. thesis, University of Sydney, 2008.

[3] J. Fiol, R. G. Pregliasco, I. Samengo, and R. O. Barrachina, An alternative classical approach to the quantum-mechanical definition of the scattering cross section, Am. J. Phys. 65, 433 (1997).

[4] L. Bao, Dynamics of student modeling: A theory, algorithms and applications to quantum physics, Ph.D. thesis, University of Maryland, 1999.
[5] R. Y. Abhang, Making introductory quantum physics understandable and interesting, Reson. J. Sci. Educ. 10, 63 (2005).

[6] D. E. Brown, Using examples and analogies to remediate misconceptions in physics: Factors influencing conceptual change, J. Res. Sci. Teach. 29, 17 (1992).

[7] H. Fischler and M. Lichtfeldt, in Research in Physics Learning: Theoretical Issues and Empirical Studies, edited by R. Duit, F. Goldberg, and H. Niedderer (IPN, Kiel, 1992), pp. 240-258.

[8] H. Fischler and M. Lichtfeldt, Modern physics and students' conceptions, Int. J. Sci. Educ. 14, 181 (1992). 
[9] K. Mannila, I. T. Koponen, and J. A. Niskanen, Building a picture of students' conceptions of wave- and particle-like properties of quantum entities, Eur. J. Phys. 23, 45 (2002).

[10] R. N. Steinberg, E. F. Redish, M. C. Wittmann, and L. Bao, Research on the Teaching and Learning of Quantum Sciences (National Association for Research in Science Teaching, Boston, 1999).

[11] L. Bao and E.F. Redish, Understanding probabilistic interpretations of physical systems: A prerequisite to learning quantum physics, Am. J. Phys. 70, 210 (2002).

[12] O. Ozcan, How do the students describe the quantum mechanics and classical mechanics?, Latin -Am. J. Phys. Educ. 4, 22 (2010) [http://www.journal.lapen.org.mx/].

[13] C. Baily and N. D. Finkelstein, Teaching and understanding of quantum interpretations in modern physics courses, Phys. Rev. ST Phys. Educ. Res. 6, 010101 (2010).

[14] M. Prosser and K. Trigwell, Understanding Learning and Teaching: The Experience in Higher Education (Society for Research into Higher Education and Open University Press, London, 1999).

[15] P. Ramsden, Improving Learning: New perspectives (Kogan Page, London, 1988).

[16] P. R. Fletcher and I. Johnston, Research on the Teaching and Learning of Quantum Sciences (National Association for Research in Science Teaching, Boston, MA, 1999).

[17] J. Falk, C. Linder, and R. L. Kung, Review of empirical studies into students' depictions of quantum mechanics, Educ. Res. Rev. (to be published).

[18] A. Mashhadi, Students' conceptions of quantum physics, in Proceedings of the International Conference on Thinking Physics for Teaching: The Case for Physics, Rome, 1994, edited by C. Bernardini (Kluwer, Dordrecht, 1996).

[19] R. V. Olsen, Introducing quantum mechanics in the upper secondary school: A study in Norway, Int. J. Sci. Educ. 24, 565 (2002).

[20] J. Falk, Students' depictions of quantum mechanics: A contemporary review and some implications for research and teaching, Ph.D. thesis, Uppsala University, 2007.

[21] S. Wuttiprom, R. Chitaree, C. Soankwan, M. Sharma, and I. Johnston, Developing a prototype conceptual survey in fundamental quantum physics, in Proceedings of the Assessment in Science Teaching and Learning Symposium (UniServe Science, Sydney, 2006), pp. 133138.

[22] F. Marton and S. Booth, Learning and Awareness (Lawrence Earlbaum, Mahwah, NJ, 1997).

[23] J. A. Browden, G. Dall'Alba, D. Laurillard, E. Martin, F. Marton, G. Masters, P. Ramsden, A. Stephanou, and E. Walsh, Displacement, velocity and frames of reference:
Phenomenographic studies of students' understanding and some implications for teaching, Am. J. Phys. 60, 262 (1992).

[24] M. Prosser, A phenomenographic study of students' intuitive and conceptual understanding of certain electrical phenomena Instr. Sci. 22, 189 (1994).

[25] M.Q. Patton, Qualitative Evaluation and Research Methods (Sage, Thousand Oaks, CA, 2002), 3rd ed.

[26] P. R. Fletcher, How students learn quantum mechanics, Master's thesis, University of Sydney, 1997.

[27] E. Cataloglu and R. W. Robinett, Testing the development of student conceptual and visualization understanding in quantum mechanics through the undergraduate career, Am. J. Phys. 70, 238 (2002).

[28] J. Falk, Developing a quantum mechanics concept inventory, Master's thesis, Uppsala University, 2004.

[29] S. B. McKagan and C.E. Wieman, Exploring student understanding of energy through the quantum mechanics conceptual survey, in Proceedings of the 2005 Physics Education Research Conference (AIP, Melville, NY, 2006).

[30] S. Goldhaber, S. Pollock, M. Dubson, P. Beale, and K. Perkins, Transforming upper-division quantum mechanics: Learning goals and assessment, in Proceedings of the 2009 Physics Education Research Conference (AIP, Melville, NY, 2009), Vol. 1179, pp. 145-148.

[31] F. Marton, Phenomenography-A research approach to investigating different understandings of reality, J. Thought 21, 29 (1986).

[32] I. D. Johnston, K. Crawford, and P. R. Fletcher, Student difficulties in learning quantum mechanics, Int. J. Sci. Educ. 20, 427 (1998).

[33] G. Ireson, A multivariate analysis of undergraduate physics students' conceptions of quantum phenomena, Eur. J. Phys. 20, 193 (1999).

[34] L. D. Carr and S. A. McKagan, Graduate quantum mechanics reform, Am. J. Phys. 77, 308 (2009).

[35] S. B. McKagan, K. K. Perkins, and C.E. Wieman, Why we should teach the Bohr model and how to teach it effectively, Phys. Rev. ST Phys. Educ. Res. 4, 010103 (2008).

[36] A. Beiser, Concepts of Modern Physics (McGraw-Hill, New York, 2002), 6th ed.

[37] R. A. Serway, Physics: For Scientists and Engineers (Thomson Burke, Fresno, CA, 2004), 6th ed.

[38] B.H. Brandsen and C. J. Joachain, Quantum Mechanics (Benjamin Cummings, San Francisco, 2000), 2nd ed.

[39] J.S. Townsend, A Modern Approach to Quantum Mechanics (University Science Books, Sausalito, CA, 2000), 2nd ed. 